\title{
Correlación entre afrontamiento y autoestima en personas con VIH de la región de los ríos, Tabasco México
}

\section{Lesli Tairi Anguiano Maldonado, Rogelio Uribe Poot, Diana Cristell Pérez Contreras}

Estudiantes de la Licenciatura en Enfermería. División Académica Multidisciplinaria de los Ríos, Universidad Juárez Autónoma de Tabasco, México.

\section{Armando Miranda de la Cruz, Sergio Quiroz Gómez}

Profesores Investigadores de la División Académica Multidisciplinaria de los Ríos, Universidad Juárez Autónoma de Tabasco, México

doi: 10.19044/esj.2017.v13n18p140 URL:http://dx.doi.org/10.19044/esj.2017.v13n18p140

\begin{abstract}
Introduction: the HIV is a disease that causes important complications of health; to achieve a good confrontation and to support a levelled autoesteem are fundamental factors for his treatment. Objective: to identify the relation between the autoesteem and the confrontation in the persons with HIV of the region of Tabasco's rivers. Methodology: quantitative, descriptive study, correlacional of transverse court, $n=64$ participants. To measure the confrontation there was in use The Scale of Strategies modified of Coping; for the autoesteem there was in use the Scale of modified Cooper Smith. Results: $48.4 \%$ belongs to the item of confrontation superior to the average; whereas $37.5 \%$ presents discharge he autoestimates. The counterfoil of correlation showed a negative significant relation of the confrontation with the autoesteem rs $=-.287$. Conclusions: the age and the years of diagnosis are determinant for the autoesteem and the confrontation; the autoesteem and the confrontation are important for the treatment and recovery of the person with HIV / AIDS.
\end{abstract}

Keywords: HIV, confrontation, autoesteem 


\section{Resumen}

Introducción: el VIH es una enfermedad que ocasiona importantes complicaciones de salud; lograr un buen afrontamiento y mantener una autoestima nivelada son factores fundamentales para su tratamiento. Objetivo: Identificar la relación entre la autoestima y el afrontamiento en las personas con VIH de la región de los ríos de Tabasco. Metodología: estudio cuantitativo, descriptivo-correlacional de corte trasversal, $n=64$ personas. Para medir el afrontamiento se utilizó la Escala de Estrategias de Coping Modificada; para la autoestima se utilizó la Escala de Cooper Smith modificada. Resultados: $48.4 \%$ pertenecen al rubro de afrontamiento superior a la media; mientras que $37.5 \%$ presentan alta autoestima. La matriz de correlación mostró una relación negativa significativa del afrontamiento con la autoestima rs $=-.287$. Conclusiones: la edad y los años de diagnóstico son determinantes para la autoestima y el afrontamiento; la autoestima y el afrontamiento son importantes para el tratamiento y recuperación de la persona con VIH/SIDA.

Palabras clave: VIH, afrontamiento, autoestima

\section{Introducción}

La Organización Mundial de la Salud (OMS, 2015) conceptualiza al Virus de la Inmunodeficiencia Humana (VIH) como un agente agresor del sistema inmunitario que altera o anula su función; dicha infección produce un deterioro progresivo con la consiguiente "inmunodeficiencia".

Según cálculos de la OMS y la Organización de Naciones Unidas contra el Síndrome de Inmunodeficiencia Adquirida (ONUSIDA), a finales de 2014 había en el mundo 36.9 millones de personas infectadas por VIH (OMS, 2015). De los cuales 1.7 millones de personas, entre ellos 33,000 niños, vivían con VIH en Latinoamérica. (ONUSIDA, 2015).

Hasta el segundo trimestre del año 2016 se tiene un conteo en México de 132,050 personas viviendo con VIH/SIDA (Centro Nacional para la Prevención y el Control del VIH/SIDA [CENSIDA], 2012). Mientras que el estado de Tabasco se había posicionado en el cuarto lugar a nivel nacional en número de casos nuevos con una tasa de 4.7 por cada 100,000 habitantes (CENSIDA, 2016).

En el municipio de Tenosique, Tabasco México se tiene hasta el primer trimestre del año 2016 un total de 132 personas infectadas con VIH/SIDA que acuden a control médico en el Centro Ambulatorio para la Prevención y Atención del SIDA y otras Infecciones de Trasmisión Sexual (CAPASITS, 2016). 
En la presente investigación se analizan dos variables de estudio: la autoestima definida como un sentimiento valorativo de nuestro ser, de nuestra manera de ser, de quienes somos nosotros, del conjunto de rasgos corporales, mentales y espirituales que configuran nuestra personalidad (Ortega Ruiz, Mínguez Vallejos \& Rodes Bravo, 2001). Por otro lado el afrontamiento definido como acción de afrontar, es decir, hacer frente a un enemigo, un peligro, una responsabilidad, entre otros (Zúñiga Barreda, 2013).

Por lo anterior se plantea la siguiente pregunta de investigación:

¿Cuál es relación entre el nivel de autoestima y el afrontamiento en personas con VIH de la región de los ríos, Tabasco?

\section{Objetivos}

\section{General}

Identificar la relación entre el nivel de autoestima y el afrontamiento de las personas con VIH de la región de los ríos, Tabasco.

Específicos

- $\quad$ Conocer las variables socio-demográficas de las personas que viven con VIH en el municipio de Tenosique, Tabasco

- Identificar el nivel de autoestima en las personas con VIH del CAPASITS del municipio de Tenosique, Tabasco

- Conocer el nivel de afrontamiento de las personas con diagnóstico confirmatorio de VIH que asisten a control en el municipio de Tenosique, Tabasco.

\section{Materiales y Métodos}

Esta investigación es de tipo cuantitativa descriptiva-correlacional de corte transversal (Pollit \& Hungler, 2000). La población de estudio se integró por un total de 132 personas diagnosticadas con VIH que acuden a control y tratamiento al CAPASITS del municipio de Tenosique de Pino Suarez, Tabasco. La muestra está constituida por 64 personas diagnosticadas con VIH. El muestreo que se utilizó fue por conveniencia.

\section{Criterios de inclusión e exclusión}

En la presente investigación se incluyeron a:

- Hombres y mujeres mayores de 18 años con diagnóstico de VIH/SIDA, que acuden a control y tratamiento en el CAPASITS de Tenosique, Tabasco (Alcántara, 2008).

Por otro lado se excluirán a:

- Pacientes con alguna complicación severa relacionada al problema de salud tales como neuroinfección, trastornos de la personalidad y depresión (Alcántara, 2008). 
- $\quad$ Pacientes con alguna alteración psico-motora o de lenguaje que imposibilite la aplicación del instrumento de recolección de datos (dislexia y/o problemas auditivos).

\section{Métodos y técnicas de recolección de datos}

El proceso de recolección de datos se realizó a través de una encuesta, la cual está integrada por tres apartados; la cual fue aplicada mediante la técnica de entrevista individualizada.

\section{Instrumentos y mediciones}

Para llevar a cabo el proceso de recolección de datos, se precisó de un instrumento que consta de las siguientes partes:

a) Consentimiento informado: en el cual se expresa de manera formal y escrita la libre voluntad de los participantes, para formar parte de la investigación en curso.

b) La cedula de datos generales: en la cual se solicitara al participante que proporcione datos tales como la edad, sexo, estado civil y tiempo de diagnóstico de VIH/SIDA

c) Instrumento para medir el nivel de afrontamiento en pacientes con VIH: Escala de Estrategias de Coping-Modificada (EEC-M) validada en Colombia por Londoño, Henao, Puerta, Posada, Arango y Aguirre (2006) y desarrollada originalmente por Chorot y Sandín (1993). La escala EEC-M de tipo likert tiene una frecuencia de respuesta que va de 1 (Nunca), 2 (Casi nunca), 3 (A veces), 4 (Frecuentemente) 5 (Casi siempre) hasta 6 (Siempre). Está compuesta por 69 ítems agrupados en 12 factores.

d) Instrumento para determinar el nivel de autoestima en pacientes con VIH: Escala de Autoestima de Cooper Smith (EAC) validada en el año 1981, consta de 58 ítems en forma de afirmaciones que valora la autoestima en 4 dimensiones que son el área personal, familiar, social y laboral.

\section{Plan de tabulación y análisis}

Para procesar los datos de esta investigación se utilizó el paquete estadístico IBM SPSS Statistics Version 22.0 para Windows 10. En el cual se analizaron los resultados de las variables cualitativas a través de distribución de frecuencias y las variables cuantitativas a través de medidas de tendencia central (media aritmética) y medidas de dispersión (desviación estándar, varianza, valor minino, valor máximo y rango).

Para conocer la relación entre las variables de estudio, se aplicó en primer lugar, la prueba de Smirnoff y Kolmogorov para determinar el coeficiente de correlación a utilizar, que en este caso fue Spearman para población anormal. 


\section{Resultados}

\section{Variables de estadística descriptiva}

A continuación se presentan las variables numéricas del presente estudio en la que se observa que la media de la edad es de $35.98(\mathrm{DE}=9.52)$, mientras que la media para el tiempo de diagnóstico es de $3.97(\mathrm{DE}=3.39)$ (ver tabla 1).

Tabla 1. Edad y tiempo de diagnóstico

\begin{tabular}{ccccc}
\hline & V. mínimo & V. máximo & Media & S \\
\hline Edad & 20 & 59 & 35.98 & 9.523 \\
Tiempo de diagnostico & 0 & 14 & 3.97 & 3.399 \\
\hline
\end{tabular}

\section{Datos sociodemográficos}

En cuanto al género de los participantes se obtuvo que $41(64.1 \%)$ pertenecen al género masculino siendo el grupo predominante en este estudio. Respecto a la ocupación del participante el grupo que predomino es el de empleado con 27 (42.2\%) del total de participantes; por otro lado el estado civil predominante en este estudio es soltero con 38 (59.4\%) del total de los participantes (ver tabla 2).

Tabla 2. Datos sociodemográficos

\begin{tabular}{cccc}
\hline Variable & Opciones & $f$ & $\%$ \\
\hline Identidad de & Femenino & 21 & 32.8 \\
genero & Masculino & 41 & 64.1 \\
& Transexual & 2 & 3.1 \\
\hline Ocupación & Labores del hogar & 17 & 26.6 \\
& Autoempleo & 5 & 7.8 \\
& Empleado & 27 & 42.2 \\
& Profesionista & 1 & 1.6 \\
& Agricultor & 5 & 7.8 \\
& Prostitución & 1 & 1.6 \\
& Obrero & 2 & 3.1 \\
& Desempleado & 6 & 9.4 \\
\hline Estado civil & Soltero & 38 & 59.4 \\
& Casado & 4 & 6.3 \\
& Unión libre & 20 & 31.3 \\
& Divorciado & 2 & 3.1 \\
\hline
\end{tabular}

\section{Variables de estudio}

$\mathrm{Al}$ analizar el instrumento EEC-M que mide el nivel de afrontamiento en las personas, se encontró que del total de la población incluida en la presente investigación $31(48.4 \%)$ tiene un nivel superior a la media de afrontamiento; mientras que $20(31.1 \%)$ poseen un nivel de afrontamiento inferior a la media y solamente $13(20.3 \%)$ poseen un nivel alto de estrategias de afrontamiento (ver tabla 3 ). 
Tabla 3. Nivel de afrontamiento en personas con VIH

\begin{tabular}{ccc}
\hline & $f$ & $\%$ \\
\hline Inferior a la media & 20 & 31.3 \\
Superior a la media & 31 & 48.4 \\
Alto & 13 & 20.3 \\
\hline Total & 64 & 100.0 \\
\hline
\end{tabular}

Por otro lado los resultados obtenidos del análisis de los datos de la EAC muestra el nivel de autoestima general de las personas entrevistadas, mostrando que $24(37.5 \%)$ tiene un nivel de autoestima alta, siendo el rubro de mayor predominancia (ver tabla 4).

Tabla 4. Nivel de autoestima en personas con VIH

\begin{tabular}{ccc}
\hline & $f$ & $\%$ \\
\hline Muy bajo & 2 & 3.1 \\
Bajo & 4 & 6.3 \\
Normal & 20 & 31.3 \\
Alto & 24 & 37.5 \\
Muy alto & 14 & 21.9 \\
\hline Total & 64 & 100.0 \\
\hline
\end{tabular}

\section{Matriz de correlación}

La matriz de correlación de Pearson mostró una relación negativa significativa del afrontamiento con la autoestima $\mathrm{rs}=-.287$. La edad guarda una relación positiva $r s=.187$ con la autoestima, mientras que los años de diagnóstico guarda una relación positiva $\mathrm{rs}=.145$ con la autoestima de los sujetos entrevistados. Por otro lado la edad guarda una relación negativa con el afrontamiento de la persona $\mathrm{rs}=-.662$ y una relación negativa entre los años de diagnóstico de la persona y el afrontamiento rs= -.153 . Mientras que los años de diagnóstico del participan guarda una relación positiva con la edad $\mathrm{rs}=.120$ (ver tabla 5$)$.

Tabla 5. Matriz de correlación de Pearson

\begin{tabular}{|c|c|c|c|c|}
\hline & Autoestima & Afrontamiento & Edad & Dx \\
\hline Autoestima & 1 & & & \\
\hline Afrontamiento & $-.287^{*}$ & 1 & & \\
\hline Edad & .187 & -.062 & 1 & \\
\hline Dx & .145 & -.153 & .120 & 1 \\
\hline
\end{tabular}

\section{Discusión}

En el estudio realizado por Gaviria, Quinceno y Vinaccia (2009), titulado "Estrategias de afrontamiento y ansiedad/depresión en pacientes diagnosticados con VIH/SIDA" en Medellín Colombia, se encontró que el sexo de predomino de los participantes fue masculino con $84.8 \%$ de los 
cuales $66.3 \%$ son empleados. De igual forma el estado civil que predomino es soltero con $77.2 \%$, mientras que la edad promedio fue de 39.4 años. Al comparar el afrontamiento como variable principal, se encontró una diferencia significativa, ya que en el estudio realizado por Gaviria, Quinceno y Vinaccia en 2009 la mayoría de los participantes (65.97\%) tiene una alta capacidad de afrontamiento, mostrando una diferencia con los resultados obtenidos en el presente.

Por otro lado Egusquiza Valera en su estudio realizado en Lima, Perú en 2010 titulado "Nivel de autoestima en pacientes con VIH-SIDA del Programa de TARGA en el Hospital Nacional Hipólito Unanue" encontró que la mayoría de los participantes pertenecen al sexo masculino (54.46\%), en un rango de edad de entre 31 y 59 años y un tiempo de diagnóstico de 1 a 4 años. En relación a la variable autoestima se encontró una diferencia marcada respecto a la presente investigación, ya que Egusquiza Valera encontró que la mayoría de los participantes tienen un nivel medio de autoestima $(55.32 \%)$, así como una relación positiva entre el nivel de autoestima y la edad de los participantes y el tiempo de diagnóstico de VIH.

\section{Conclusión}

Con la realización del presente estudio de investigación se puede establecer que el nivel de afrontamiento de las personas entrevistadas se encuentra en el rubro de superior a la media; mientras que la autoestima de las personas incluidas en este estudio se encuentra en un nivel alto, lo que ha decir de diversos autores, representa una desviación importante de la autoestima, ya que ambos extremos de la escala representan un problema de salud mental.

De igual forma se puede afirmar que la relación entre autoestima y el afrontamiento de los pacientes con VIH que acuden a control y tratamiento en el CAPASITS del municipio de Tenosique, Tabasco es negativa significativa, y factores como la edad y el tiempo de diagnóstico influyen de forma directa sobre estas dos variables, modificándolas parcial o totalmente. Dichas variables representan un aspecto importante y trascendental en el apego al tratamiento de personas con VIH y por consecuencia al logro de objetivos terapéuticos establecidos conjuntamente entre profesionales y personas; asegurando de este modo la mejora en la situación de salud biopsicosocial.

\section{References:}

1. Alcatara, Z.N. (2008). Sintomatología depresiva y adhesión al tratamiento en pacientes con VIH. (Tesis inédita de maestría) Pontificia Universidad Católica de Perú. 
2. Centro Nacional para la Prevención y el Control del VIH y el SIDA (2016). Vigilancia Epidemiológica de casos de VIH/SIDA en México. Registro Nacional de Casos de SIDA, actualización al Segundo trimestre de 2016. Disponible en: http://www.censida.salud.gob.mx/descargas/epidemiologia/RN_2do_ trim_2016.pdf

3. Egusquiza, V.E. (2010). Nivel de Autoestima en pacientes con VIHSIDA del programa de TARGA en el Hospital Nacional Hipólito Unanue. (Tesis inédita de maestría). Universidad Nacional Mayor de San Marcos.

4. Hernández, S. R., Fernández, C. C. \& Baptista, L. P. (2006). Metodología de la investigación. $4^{a}$ edición. México: McGraw-Hill.

5. Milena, G.A., Quinceno, J. \& Vinaccia, S. (2001). Estrategias de afrontamiento y Ansiedad-Depresión en pacientes diagnosticados con VIH/SIDA. Terapia Psicológica. Volumen (27). pp. 5-13.

6. Organización de las Naciones Unidas contra el Síndrome de Inmunodeficiencia Adquirida (2015). Situación de la epidemia del VIH en América Latina. Disponible en: http://onusidalac.org/1/index.php/situacion-de-la-epidemia-del-vihen-america-latina-infografia

7. Organización mundial de la salud (2015). Nota descriptiva No. 360. Disponible en: http://www.who.int/mediacentre/factsheets/fs360/es/

8. Ortega, R.P., Mínguez, V.R. \& Rodes, B.M. (2001). Autoestima: un nuevo concepto y su medida. Teorías de la Educación. Volumen (12). pp. 45-66.

9. Polit, D.F. \& Hungler. BP. (2000). Investigación científica en ciencias de la salud: principios y métodos. $6^{\mathrm{a}}$ edición. México: McGraw-Hill

10. Zúñiga, B.F. (2005). Afrontamiento: visión general. Revista Chilena de Salud. Volumen (15) pp. 13-18. 